EMERGENCE OF VACCINE-DERIVED POLIOVIRUSES DURING EBOLA VIRUS DISEASE OUTBREAK IN GUINEA, 2014-2015

M.D. Fernandez-Garcia ${ }^{1}$, M. Majumdar ${ }^{2}$,

O. Kebe' ${ }^{1}$, A.D. Fall ${ }^{1}$, M. Kone ${ }^{2}$, M. Kande ${ }^{2}$,

M. Dabo ${ }^{2}$, M.S. Sylla ${ }^{2}$, D. Sompare ${ }^{2}$, W. Howard ${ }^{4}$,

O. Faye ${ }^{1}$, J. Martin ${ }^{3}$, K. Ndiaye ${ }^{1}$

${ }^{1}$ Institut Pasteur, Dakar, Senegal; ${ }^{2}$ Ministry of Health, Conakry, Republic of Guinea; ${ }^{3}$ National Institute for Biological Standards and Control, Potters Bar, United Kingdom;

${ }^{4}$ National Institute for Communicable Diseases, Johannesburg, South Africa

From December 2013 to May 2016, 3.351 laboratory-confirmed cases of EVD occurred in Guinea, resulting in 2.083 deaths and reaching a peak of 509 confirmed cases in October 2014. During this outbreak, 13 type 2 circulating vaccine-derived polioviruses (cVDPVs) were isolated from 6 polio patients and 7 healthy contacts. To clarify the genetic properties of cVDPVs and their emergence, we combined epidemiologic and virologic data for polio cases in Guinea.

Patients with paralytic poliomyelitis were identified through Guinea's AFP surveillance system according to WHO guidelines. During September 2015-December 2016, additional fecal samples were collected from contacts of most AFP patients.

Polioviruses were isolated from fecal samples according to WHO standard procedures and subjected to intratypic differentiation by reverse transcription PCR targeting the VP1 region. Typing of non-polio enterovirus isolates was performed by RT-snPCR targeting part of the 3'-VP3 and the 5'-VP1 regions. Isolates with discordant intratypic differentiation results were sent to the National Institute for Communicable Diseases, Johannesburg, South Africa, for entire VP1 sequencing according to WHO guidelines

To assess epidemiologic factors associated with the outbreak of Ebola Virus Disease (EBV), field investigations were conducted during December 17-28, 2015, in Siguiri and Kankan Prefectures, Guinea.

In September 2014, a case of laboratory-confirmed type $2 \mathrm{cVDPV}$ infection was identified in Guinea (Siguiri Prefecture). During October 2014-March 2015, collection of fecal samples from AFP patients in Guinea was interrupted because of the outbreak of EVD. On September 4, 2015, type $2 \mathrm{cVDPV}$ was isolated from a fecal sample of a child living in the Kankan region of Guinea. Subsequently, type 2 cVDPV isolates were recovered from 5 other AFP patients and 7 healthy contacts. The 7 healthy type $2 \mathrm{cVDPV}$-positive contacts were epidemiologically linked to 3 of the AFP case-patients. Most (12/13) poliovirus-positive case-patients were incompletely vaccinated children. All 13 type 2 cVDPV strains were isolated from persons in the Kankan region in eastern Guinea, most (12/13) persons were from Siguiri Prefecture. During the first semester of 2015, the coverage of routine OPV3 vaccination in Siguiri Prefecture was $31 \%$. In 2014, the official national OPV3 routine coverage in Guinea was $42 \%$.

All 13 VDPV isolates showed discordant intratypic differentiation results and were further characterized by sequencing the VP1 capsid-coding region. All isolates diverged $>0.6 \% \mathrm{nt}$ from the type 2 OPV strain, and classified as type 2 VDPVs. The 13 strains clustered in a monophyletic group with a high (96\%) bootstrap value
Deviation of public health resources to the Ebola outbreak disrupted polio vaccination programs and surveillance activities, which fueled the spread of neurovirulent VDPVs in an area of low vaccination coverage and immunity. Genetic properties of cVDPVs were consistent with their capacity to cause paralytic disease in humans and capacity for sustained person-to-person transmission. Circulation ceased when coverage of oral polio vaccine increased.

A polio outbreak in the context of the Ebola virus disease outbreak highlights the need to consider risks for polio emergence and spread during complex emergencies and urges awareness of the challenges in polio surveillance, vaccination, and diagnosis.

3.16 doi: 10.15789/2220-7619-2018-4-3.16

\section{ENTEROVIRUS INFECTION IN THE RUSSIAN FEDERATION IN 2008-2018}

L.N. Golitsyna, V.V. Zverev, S.G. Fomina, D.V. Sozonov, N.A. Novikova

Blokhina Nizhny Novgorod Research Institute of Epidemiology and Microbiology, Nizhny Novgorod, Russia

In the XXI century in Europe (including Russia), Asia and the Pacific region occur a significant increase in the incidence of enterovirus infection (EVI). In Russia, the rates of registered incidence of EVI and enteroviral meningitis (ASM) are included in official statistical reporting in 2006.

The Reference Center for Monitoring EVI, established in 2008, studies the molecular epidemiology of enteroviruses (EVs) and EVI. The work is carried out in close cooperation with the Directorates of Rospotrebnadzor and the Centers for Hygiene and Epidemiology in the subjects of the Russian Federation (RF) and the scientific centers.

EV-positive samples from 7940 patients with various clinical manifestations and 656 environmental objects collected in different regions of Russia in 2007-2018 were studied using partial sequencing of the region of the VP1 genome.

Nonpolio EVs were typed in 4323 cases. 53 types of viruses were identified: sp. Enterovirus A: CVA2-8, CVA10, CVA14, CVA16, EV-A71, EV-A76, EV-A120; $s p$. Enterovirus B: CVB1-5, CVA9, E1-7, E9, E11, E13-21, E25, E29, E30, E31, E33; sp. Enterovirus C: CVA1, CVA13, CVA17, CVA19, CVA20-22, CVA24, EV-C99, EV-C113, EV-C116. Annually, about 30 types of nonpolio EVs were detected, and many types of viruses were characterized by the presence of several simultaneously circulating genovariants.

During the observation period, all peaks of enteroviral meningitis incidence in the RF registered in 2008-2009, 2013 and 2016-2017, were associated with E30. The E30 of the genotype eC2 circulated in 2007-2009. In 2013, E30 of genotype $h$ has become more active and continues to circulate until now. Among the causative agents of ASM, in addition to E30, during this period the most active viruses were E9, E6, CVA9, CVB2-5 and other members of the sp. Enterovirus B.

The increase in the incidence of HFMD in the Russia was associated with an increase in the circulating activity of viruses of the sp. Enterovirus A (primarily CVA6 of new genotype), which began in 2010 .

The current situation in the RF on the incidence of EVI requires continuous molecular monitoring of the circulation of epidemic variants of enteroviruses, and in case of its aggravation, the development of specific means of prevention. 